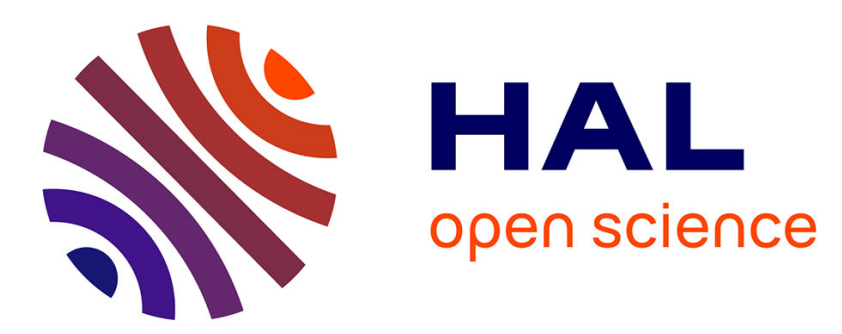

\title{
A Self-Triggered Control Based on Convex Embeddings for Perturbed LTI Systems
}

Christophe Fiter, Laurentiu Hetel, Wilfrid Perruquetti, Jean-Pierre Richard

\section{To cite this version:}

Christophe Fiter, Laurentiu Hetel, Wilfrid Perruquetti, Jean-Pierre Richard. A Self-Triggered Control Based on Convex Embeddings for Perturbed LTI Systems. 9th IFAC Symposium on Nonlinear Control Systems, Sep 2013, Toulouse, France. pp.335-340, 10.3182/20130904-3-FR-2041.00176 . hal00849840

\section{HAL Id: hal-00849840 \\ https://hal.inria.fr/hal-00849840}

Submitted on 7 Sep 2015

HAL is a multi-disciplinary open access archive for the deposit and dissemination of scientific research documents, whether they are published or not. The documents may come from teaching and research institutions in France or abroad, or from public or private research centers.
L'archive ouverte pluridisciplinaire HAL, est destinée au dépôt et à la diffusion de documents scientifiques de niveau recherche, publiés ou non, émanant des établissements d'enseignement et de recherche français ou étrangers, des laboratoires publics ou privés. 


\title{
A Self-Triggered Control Based on Convex Embeddings for Perturbed LTI Systems *
}

\author{
C. Fiter $^{*}$ L. Hetel ${ }^{* *}$ W. Perruquetti ${ }^{* *, * *}$ J.-P. Richard ${ }^{* *, * * *}$ \\ * Department of Electrical Engineering - Systems, Tel-Aviv University, \\ Tel-Aviv,69978, Israel (email: christophe.fiter@centraliens-lille.org) \\ ** Laboratoire d'Automatique, Génie Informatique et Signal (CNRS \\ UMR 8219), École Centrale de Lille, 59651 Villeneuve d'Ascq, France \\ *** NON-A Project team, INRIA Lille-Nord Europe, France
}

\begin{abstract}
In this work, we present a novel self-triggered control which aims at decreasing the number of sampling instants for the state feedback control of perturbed linear time invariant systems. The approach is based on convex embeddings that allow for designing a state-dependent sampling function guaranteeing the system's exponential stability for a desired decay-rate and norm-bounded perturbations. One of the main contributions of this paper is an LMI based algorithm that optimizes the choice of the Lyapunov function so as to enlarge the lower-bound of the sampling function while taking into account both the perturbations and the decay-rate. The advantages of the approach are illustrated with a numerical example from the literature.
\end{abstract}

Keywords: networked control systems, sampled-data systems, self-triggered control, linear time-invariant, linear matrix inequality, Lyapunov, convex embedding

\section{INTRODUCTION}

In the past decade, Networked Control Systems have become an important center of attention of researchers in control theory. These systems present numerous advantages, such as reduced wiring, plug and play devices, increased agility, and ease of maintenance. However, because they are often required to share a limited amount of resources, they generate fluctuations in the sampling intervals, which may result in unstable behaviours. This brings up new theoretical challenges.

In order to take into consideration these new difficulties, several works have studied the stability of sampled-data systems with time-varying sampling (Fujioka [2009], Fridman [2010], Seuret [2012], Liu and Fridman [2012], Fiter et al. [2012]).

More recently, another research direction has emerged, consisting in controlling dynamically the sampling instants so as to reduce the processor and/or network loads while ensuring the desired control performances. Two main approaches can be found in the literature:

- The first approach is the event-triggered control (Tabuada [2007], Lunze and Lehmann [2010], Heemels et al. [2011], Postoyan et al. [2011]), in which intelligent sensors send information to the controller when special events occur (for

\footnotetext{
^ This work was partially supported by Israel Science Foundation (grant No 754/10), by the Ministry of Higher Education and Research, Nord-Pas de Calais, and FEDER through the 'CPER 2007-2013', by the European Community's Seventh Framework Programme (FP7/2007-2013) under grant agreement $\mathrm{n}^{\circ}$ 257462: HYCON2 Network of Excellence "Highly-Complex and Networked Control Systems", and by the INTERREG IV A 2 Mers Seas Zeen Cross-border Cooperation Program 2007-2013 under SYSIASS 6-20 project.
}

example when the system's state leaves some boundary around the equilibrium point). In most works from the literature, this approach requires a dedicated hardware. - The second approach is the self-triggered control (Mazo Jr. et al. [2010], Anta and Tabuada [2010], Wang and Lemmon [2010]), which emulates event-triggered control without dedicated hardware, by computing at each sampling instant a lower-bound of the next admissible sampling interval. In general in these works, no method is given to compute the Lyapunov function so as to optimize the lower-bound of the sampling intervals (i.e. in the worst case, independently of the system's state).

Still more recently, in Fiter et al. [2012], the authors proposed a third dynamic sampling control approach, statedependent sampling, which consists in designing the statedependent sampling function (i.e. the self-triggered controller) offline, thanks to a mapping of the state space and linear matrix inequalities (LMIs). An advantage was that these LMIs could be used for optimizing the Lyapunov function, thus solving the issue mentioned previously regarding regular self-triggered control approaches. However, only ideal linear time invariant (LTI) systems were considered.

In the present work, we present a novel self-triggered control scheme in the case of LTI systems with unknown, exogenous, state-bounded perturbations. The communication links are assumed to react instantaneously (i.e. there is no communication delay). We guarantee the system's exponential stability for a desired decay-rate using Lyapunov stability conditions and convexification arguments. One of the main contributions and advantages is the optimization of the Lyapunov function, taking into account both the effects of the sampling and the perturbation. It optimizes 
the lower-bound of the sampling intervals for the proposed self-triggered control scheme.

The paper is organized as follows. First, we state the problem in Section 2 and propose the main stability result in Section 3. Then, we design the self-triggered controller in Section 4. Finally, simulation results are shown in Section 5 before concluding in Section 6 . All the proofs are given in the Appendix, along with intermediate results.

Notations: $\mathbb{R}_{+}=\{\lambda \in \mathbb{R}, \lambda \geq 0\}, \mathbb{R}^{*}=\{\lambda \in \mathbb{R}, \lambda \neq 0\}$, $\mathbb{N}^{*}=\{n \in \mathbb{N}, n \neq 0\}$, and $\mathbb{R}_{+}^{*}=\mathbb{R}_{+} \backslash\{0\} . \lambda_{\max }(M)$ denotes the largest eigenvalue of a symmetric matrix $M \in$ $\mathbb{R}^{n \times n} \cdot \mathbb{S}_{n}^{+}$(resp. $\mathbb{S}_{n}^{+*}$ ) is the set of positive (resp. positive definite) symmetric matrices $P \succeq 0$ (resp. $P \succ 0$ ) in $\mathbb{R}^{n \times n}$. The symmetric elements of a symmetric matrix are denoted by $* .\|$.$\| is the Euclidean norm on \mathbb{R}^{n}$.

\section{PROBLEM STATEMENT}

\subsection{System description}

Consider the perturbed LTI system

$$
\dot{x}(t)=A x(t)+B u(t)+E w(t), \forall t \in \mathbb{R}_{+},
$$

where $x: \mathbb{R}_{+} \rightarrow \mathbb{R}^{n}, u: \mathbb{R}_{+} \rightarrow \mathbb{R}^{n_{u}}$, and $w: \mathbb{R}_{+} \rightarrow$ $\mathbb{R}^{n_{w}}$ represent respectively the system state, the control function, and the exogenous disturbance. The matrices $A$, $B$, and $E$ are constant with appropriate dimensions.

The control is a piecewise-constant state feedback

$$
u(t)=-K x\left(t_{k}\right), \forall t \in\left[t_{k}, t_{k+1}\right), \forall k \in \mathbb{N},
$$

where the gain $K$ is fixed and such that $A-B K$ is Hurwitz.

The sampling instants $t_{k}$ are defined by the self-triggered control law

$$
t_{k+1}=t_{k}+\tau\left(x\left(t_{k}\right)\right), \forall k \in \mathbb{N},
$$

where $\tau: \mathbb{R}^{n} \rightarrow \mathbb{R}_{+}$is a state-dependent sampling function to be designed.

The disturbance is assumed to be state-bounded:

$$
\exists W \geq 0,\|w(t)\|^{2} \leq W\left\|x\left(t_{k}\right)\right\|^{2}, \forall t \in\left[t_{k}, t_{k+1}\right), \forall k \in \mathbb{N} .
$$

Such a perturbation can represent model uncertainties, local nonlinearities, or some kind of measurement noises.

We denote by $\mathcal{S}$ the closed-loop system $\{(1),(2),(3),(4)\}$.

\subsection{Objectives}

Our goal is to design a sampling function $\tau$ as large as possible and with a maximal lower-bound, while ensuring the exponential stability of $\mathcal{S}$ for a desired decay-rate $\alpha$, also called $\alpha$-stability (i.e. such that there exists a scalar $\gamma$ for which all trajectories satisfy $\|x(t)\| \leq \gamma \mathrm{e}^{-\alpha t}\left\|x_{0}\right\|$ for any initial condition $x_{0}$ ).

To this aim, we use the well known Lyapunov exponential stability property:

Proposition 1. (See Fridman [2010] for example) Consider a scalar $\alpha>0$ and a quadratic Lyapunov candidate function $V(x)=x^{T} P x, \forall x \in \mathbb{R}^{n}$, with $P \in \mathbb{S}_{n}^{+*}$. If the condition

$$
\dot{V}(x)+2 \alpha V(x) \leq 0
$$

is satisfied for all trajectories of $\mathcal{S}$, then the system is globally $\alpha$-stable.
Our objectives can then be formulated as:

Objective 1: Given a quadratic Lyapunov function $V$, design a lower-bound approximation of the optimal sampling function $\tau_{\text {opt }}^{V}(x)=\max \tau(x)$ such that (5) holds.

Objective 2: Find a quadratic Lyapunov function $V$ such that there exists a sampling function $\tau$ satisfying (5) with a minimum value $\tau^{*}=\inf _{x \in \mathbb{R}^{n}} \tau(x)$ as large as possible.

\section{MAIN STABILITY RESULT}

In this section, we provide sufficient stability conditions that depend on the time variable $\sigma \triangleq t-t_{k}$ and on the sampled-state $x \triangleq x\left(t_{k}\right)$.

The following result is obtained by bounding the effects of the perturbations on the system's behaviour thanks to (4), by using the well known inequality (Cao et al. [1998])

$$
x^{T} y+y^{T} x \leq \varepsilon^{-1} x^{T} x+\varepsilon y^{T} y,
$$

which is satisfied for any vectors $x$ and $y$ of same dimension, and any scalar $\varepsilon>0$.

Theorem 2. Consider a tuning parameter $\varepsilon>0$, scalars $\alpha>0$ and $W \geq 0$, and a sampling function $\tau: \mathbb{R}^{n} \rightarrow \mathbb{R}_{+}$. Then, the system $\mathcal{S}$ is globally $\alpha$-stable if there exist a matrix $P \in \mathbb{S}_{n}^{+*}$ and scalars $\eta \geq 0$ and $\mu \geq 0$ such that

$$
\left(1+\varepsilon^{-1}\right) E^{T} P E \preceq \eta I, Q_{5} \preceq \mu I,
$$

and

$$
x^{T} \Pi(\sigma) x \leq 0, \forall x \in \mathbb{R}^{n}, \forall \sigma \in[0, \tau(x)],
$$

with

$$
\Pi(\sigma)=\Lambda(\sigma)^{T} Q_{1} \Lambda(\sigma)+\Lambda(\sigma)^{T} Q_{2}+Q_{2}^{T} \Lambda(\sigma)+Q_{3}+Q_{4}(\sigma),
$$

$$
\Lambda(\sigma)=I+\int_{0}^{\sigma} \mathrm{e}^{s A} d s(A-B K),
$$

$$
\begin{aligned}
& Q_{1}=(1+2 \alpha \varepsilon)\left(A^{T} P+P A\right)+\varepsilon A^{T} P A \\
&+2\left(\alpha+\varepsilon+2 \varepsilon \alpha^{2}\right) P \\
& Q_{2}=-\left(I+\varepsilon(A+2 \alpha I)^{T}\right) P B K \\
& Q_{3}= \varepsilon K^{T} B^{T} P B K+W \eta I \\
& Q_{4}(\sigma)=\sigma W \mu \lambda_{\max }\left(E^{T} E\right) f_{A}(\sigma) I \\
& Q_{5}=A^{T} P+P A+\varepsilon^{-1} A^{T} P A+\left(1+2 \alpha+\varepsilon^{-1}\right) P,
\end{aligned}
$$
and

$$
f_{A}(\sigma)=\left\{\begin{array}{l}
\frac{1}{\lambda_{\max }\left(A+A^{T}\right)}\left(\mathrm{e}^{\lambda_{\max }\left(A+A^{T}\right) \sigma}-1\right) \\
\text { if } \lambda_{\max }\left(A+A^{T}\right) \neq 0 \\
\sigma \text { otherwise. }
\end{array}\right.
$$

Remark 1: Since $A-B K$ is assumed to be Hurwitz (i.e. the LTI system (1) without perturbation is stable with the continuous state feedback $u(t)=-K x(t)$ ), one can show that there always exist parameters $P, \eta$ and $\mu$, such that the conditions $(7)$ are satisfied and such that $\Pi(0) \prec 0$, provided that the tuning parameter $\varepsilon$, the decay-rate $\alpha$, and the perturbations upper-bound $W$ are small enough (if $\varepsilon, \alpha$, and $W$ are close to 0 , then $\Pi(0) \simeq(A-B K)^{T} P+$ $P(A-B K))$. With such parameters, we can thus find a sampling function $\tau$ satisfying the stability conditions of Theorem 2, which is lower-bounded by a strictly positive scalar. An algorithm enlarging that lower-bound will be provided in the next section. 


\section{SELF-TRIGGERED CONTROLLER DESIGN}

The self-triggered control scheme we propose in this paper is based on the stability conditions from Theorem 2, which involve a few LMIs (7) (considering that $\varepsilon$ is a tuning parameter) as well as the more complex set of conditions: $x^{T} \Pi(\sigma) x \leq 0, \forall x \in \mathbb{R}^{n}, \forall \sigma \in[0, \tau(x)]$.

These stability conditions can not be checked "as is" in real time, since they involve an infinite number of inequalities regarding the time variable $\sigma$. Therefore, in order to obtain checkable conditions, we propose to use:

- A discretization of the time interval, with a step $\Delta \in \mathbb{R}_{+}^{*}$ (which can be either chosen by the user or imposed by the system);

- Convex embeddings around the matrix function $\Pi$ over every time interval $[j \Delta,(j+1) \Delta]$ (with $j \in \mathbb{N}$ ), so as to guarantee the desired performances between any two successive discrete instants. Indeed, since the matrix function $\Pi$ is continuous, then for any $j \in \mathbb{N}$ it is possible to design a convex polytope with a finite number of vertices $\Pi_{i}^{(j)}(\Delta)(i \in\{0, \cdots, N\})$ such that for all $x \in \mathbb{R}^{n}$,

$$
\begin{gathered}
\left(x^{T} \Pi_{i}^{(j)}(\Delta) x \leq 0, \forall i \in\{0, \cdots, N\}\right) \\
\Downarrow \\
\left(x^{T} \Pi(\sigma) x \leq 0, \forall \sigma \in[j \Delta,(j+1) \Delta]\right) .
\end{gathered}
$$

\subsection{Convex embedding design based on Taylor polynomials}

Here, we adapt the result from Hetel et al. [2006] to design a convex embedding satisfying (13). The approach is based on an $N$-order Taylor series approximation of $\Pi$.

The vertices are defined for $i \in\{0, \cdots, N\}$ and $j \in \mathbb{N}$ as

with

$$
\Pi_{i}^{(j)}(\Delta)=\hat{\Pi}_{i}^{(j)}(\Delta)+\nu^{(j)}(\Delta) I,
$$

$$
\begin{gathered}
\hat{\Pi}_{i}^{(j)}(\Delta)=\sum_{k=0}^{i} \Psi_{k}^{(j)}(\Delta) \Delta^{k} \\
\Psi_{0}^{(j)}(\Delta)=\Gamma_{1}^{(j)}(\Delta)^{T} Q_{1} \Gamma_{1}^{(j)}(\Delta) \\
+\Gamma_{1}^{(j)}(\Delta)^{T} Q_{2}+Q_{2}^{T} \Gamma_{1}^{(j)}(\Delta)+Q_{3}+L_{0}^{(j)}(\Delta) \\
\Psi_{1}^{(j)}(\Delta)=\Gamma_{2}^{(j)}(\Delta)^{T}\left(Q_{1} \Gamma_{1}^{(j)}(\Delta)+Q_{2}\right) \\
+\left(\Gamma_{1}^{(j)}(\Delta)^{T} Q_{1}^{T}+Q_{2}^{T}\right) \Gamma_{2}^{(j)}(\Delta)+L_{1}^{(j)}(\Delta) \\
\Psi_{k \geq 2}^{(j)}(\Delta)=\Gamma_{2}^{(j)}(\Delta)^{T} \frac{\left(A^{k-1}\right)^{T}}{k !}\left(Q_{1} \Gamma_{1}^{(j)}(\Delta)+Q_{2}\right) \\
+\left(\Gamma_{1}^{(j)}(\Delta)^{T} Q_{1}^{T}+Q_{2}^{T}\right) \frac{A^{k-1}}{k !} \Gamma_{2}^{(j)}(\Delta) \\
+\Gamma_{2}^{(j)}(\Delta)^{T}\left(\sum_{i=1}^{k-1} \frac{\left(A^{i-1}\right)^{T}}{i !} Q_{1} \frac{A^{k-i-1}}{(k-i) !}\right) \Gamma_{2}^{(j)}(\Delta) \\
+L_{k}^{(j)}(\Delta),
\end{gathered}
$$

and

$$
\begin{aligned}
& \Gamma_{1}^{(j)}(\Delta)=I+N_{j}(\Delta)(A-B K) \\
& \Gamma_{2}^{(j)}(\Delta)=N_{j}^{\prime}(\Delta)(A-B K), \\
& N_{j}(\Delta)=\int_{0}^{j \Delta} \mathrm{e}^{A s} d s, N_{j}^{\prime}(\Delta)=A N_{j}(\Delta)+I .
\end{aligned}
$$

If $\lambda_{\max }\left(A+A^{T}\right)=0$, the matrices $L_{k}(j)$ are defined as

$$
L_{0}^{(j)}(\Delta)=W \mu \lambda_{\max }\left(E^{T} E\right)(j \Delta)^{2} I,
$$

$$
\begin{aligned}
& L_{1}^{(j)}(\Delta)=2 W \mu \lambda_{\max }\left(E^{T} E\right) j \Delta I, \\
& L_{2}^{(j)}(\Delta)=W \mu \lambda_{\max }\left(E^{T} E\right) I, \\
& L_{k \geq 3}^{(j)}(\Delta)=0 .
\end{aligned}
$$

Otherwise, if $\lambda_{\max }\left(A+A^{T}\right) \neq 0$, they are defined as

$$
\begin{gathered}
L_{0}^{(j)}(\Delta)=W \mu \frac{\lambda_{\max }\left(E^{T} E\right)}{\lambda_{\max }\left(A+A^{T}\right)} j \Delta\left(\mathrm{e}^{\lambda_{\max }\left(A+A^{T}\right) j \Delta}-1\right) I, \\
L_{1}^{(j)}(\Delta)=W \mu \frac{\lambda_{\max }\left(E^{T} E\right)}{\lambda_{\max }\left(A+A^{T}\right)} \\
\left(\mathrm{e}^{\lambda_{\max }\left(A+A^{T}\right) j \Delta}\left(1+j \Delta \lambda_{\max }\left(A+A^{T}\right)\right)-1\right) I, \\
L_{k \geq 2}^{(j)}(\Delta)=W \mu \frac{\lambda_{\max }\left(E^{T} E\right)}{\lambda_{\max }\left(A+A^{T}\right)} \mathrm{e}^{\lambda_{\max }\left(A+A^{T}\right) j \Delta} \\
\left(j \Delta \frac{\left(\lambda_{\max }\left(A+A^{T}\right)\right)^{k}}{k !}+\frac{\left(\lambda_{\max }\left(A+A^{T}\right)\right)^{k-1}}{(k-1) !}\right) I .
\end{gathered}
$$

Finally,

$$
\nu^{(j)}(\Delta) \geq \max _{\sigma \in[j \Delta,(j+1) \Delta]} \lambda_{\max }\left(\Pi(\sigma)-\sum_{k=0}^{N} \Psi_{k}^{(j)}(\Delta)(\sigma-j \Delta)^{k}\right) .
$$

Remark 2: The matrices $\Psi_{k}^{(j)}(\Delta)$ defined in (16) are the coefficients of the Taylor polynomial of $\Pi$ over $[j \Delta,(j+$ 1) $\Delta$ ]. The constants $\nu^{(j)}(\Delta)$ defined in (20) represent upper-bounds of the Taylor series approximation error over each time intervals $[j \Delta,(j+1) \Delta]$.

Theorem 3. Consider $\Delta \in \mathbb{R}_{+}^{*}, j \in \mathbb{N}$, and $x \in \mathbb{R}^{n}$. The polytope vertices $\Pi_{i}^{(j)}(\Delta)$ defined in (14) satisfy (13): if $x^{T} \Pi_{i}^{(j)}(\Delta) x \leq 0$ for all $i \in\{0, \cdots, N\}$, then $x^{T} \Pi(\sigma) x \leq 0$ for all $\sigma \in[j \Delta,(j+1) \Delta]$.

\subsection{Design of the sampling function $\tau$ for given parameters}

In this subsection, we consider that the parameters $P \in$ $\mathbb{S}_{n}^{+*}, \eta \geq 0, \mu \geq 0$, and $\varepsilon>0$ satisfying (7) are given.

Then, using Theorem 3 , it is possible to design a lowerbound estimation $\tau$ of the maximal state-dependent sampling function satisfying the stability condition (8) from Theorem 2 as:

$$
\tau(x)=j(x) \Delta,
$$

with

$$
j(x)=\min \left\{j \in \mathbb{N} \mid \exists i \in\{0, \cdots, N\}, x^{T} \Pi_{i}^{(j)}(\Delta) x>0\right\} .
$$

Remark 3: It is important to guarantee that $j(x)>0$ (and thus $\tau(x)>0$ ) for any state $x \in \mathbb{R}^{n}$. To this aim, one may simply check if the inequality $\Pi_{i}^{(0)}(\Delta) \preceq 0$ is satisfied for all $i \in\{0, \cdots, N\}$. If this is not the case, it may be that the discretization step time $\Delta$ is too large, or that the tuning parameter $\varepsilon$, the decay-rate $\alpha$, or the perturbations upper-bound $W$ are also too large (see Remark 1). In the following subsection, we will show how to design all these parameters so as to optimize the lower-bound of the sampling function.

Remark 4: Note that the matrices $\Pi_{i}^{(j)}(\Delta)$ can be computed offline. The number of online computations 
required to perform the self-triggered control scheme (i.e. to compute $j(x))$ is upper-bounded by $n(n+1)(N+$ 1) $\frac{\tau(x)}{\Delta}$ multiplications and $(n+1)(n-1)(N+1) \frac{\tau(x)}{\Delta}$ additions. The online complexity is thus $O\left(\frac{\tau(x)}{\Delta} N n^{2}\right)$, which is comparable to the one obtained in the selftriggered control scheme from Mazo Jr. et al. [2010] for example. Note that choosing a larger step $\Delta$ will reduce the number of computations, but will end in a loss of precision for the sampling function (21). Therefore, a trade-off has to be made between precision and online complexity.

\subsection{Optimization of the parameters (maximization of the lower-bound $\tau^{*}$ of the sampling function)}

In this subsection, we propose an LMI-based algorithm that computes the different parameters involved in the selftriggered control scheme (the Lyapunov matrix $P \in \mathbb{S}_{n}^{+*}$ and the additional parameters $\varepsilon>0, \eta \geq 0$, and $\mu \geq 0$ ) so as to optimize the lower-bound of the sampling function (21). It is based on the following property:

Theorem 4. Consider a tuning parameter $\varepsilon>0$, a timestep $\Delta>0$, and scalars $\alpha>0$ and $W \geq 0$. Consider a matrix $P \in \mathbb{S}_{n}^{+*}$, scalars $\eta \geq 0$ and $\mu \geq 0$, and a sampling function $\tau: \mathbb{R}^{n} \rightarrow \mathbb{R}_{+}$such that the conditions from Theorem 2 hold. If there exists an integer $j^{*} \in \mathbb{N}^{*}$ such that the matrix inequalities $\Pi_{i}^{(j)}(\Delta) \preceq 0$ are satisfied for all $i \in\{0, \cdots, N\}$ and all $j \in\left\{0, \cdots, j^{*}-1\right\}$, then the system $\mathcal{S}$ is globally $\alpha$-stable with the sampling function $\bar{\tau}: \mathbb{R}^{n} \rightarrow \mathbb{R}_{+}$defined as $\bar{\tau}(x)=\max \left(\tau(x), j^{*} \Delta\right)$.

The conditions $\Pi_{i}^{(j)}(\Delta) \preceq 0$ in Theorem 4 depend from the parameters $P, \eta, \mu$, and $\varepsilon$. Therefore, it becomes clear that maximizing the sampling function lower-bound reduces to searching the parameters that maximize $\tau^{*} \triangleq j^{*} \Delta$. This can be done as follows.

\section{Algorithm:}

Step 1: In this step, we consider the polytopic description (14) with upper-bounds on the estimation errors $\nu^{(j)}(\Delta)=$ 0 . Then, for a given $\varepsilon>0$, the search for $P, \eta$ and $\mu$ maximizing $\tau^{*}$ in Theorem 4 reduces to an LMI problem (from (11) and (14) to (19), we can see that the matrices $\Pi_{i}^{(j)}(\Delta)$ are linear in $P, \eta$ and $\mu$ ), which can be solved using LMI solvers. It is possible to find the appropriate parameter $\varepsilon$ with a linear search algorithm.

Step 2: Next, we compute the value of the upper-bounds $\overline{\nu^{(j)}(\Delta)}$ and of the matrices $\Pi_{i}^{(j)}(\Delta)$ that correspond to the obtained parameters $P, \eta, \mu$, and $\varepsilon$.

Step 3: Finally, the lower-bound of the sampling function can be designed as

with

$$
\tau^{*}=j^{*} \Delta
$$

$$
j^{*}=\min \left\{j \in \mathbb{N} \mid \exists i \in\{0, \cdots, N\}, \Pi_{i}^{(j)}(\Delta) \succ 0\right\} .
$$

This parameter optimization presents several advantages, among which the guarantee of a large lower-bound $\tau^{*}=$ $j^{*} \Delta$ for the sampling intervals, and the reduction of the online complexity, since it not necessary anymore to check the inequalities $x^{T} \Pi_{i}^{(j)}(\Delta) x>0$ for $j \in\left\{0, \cdots, j^{*}-1\right\}$ when computing $j(x)$ in $(22)$.

\section{NUMERICAL EXAMPLE}

Consider the Batch Reactor system from Mazo Jr. et al. [2009] with some additional perturbation:

$$
\begin{aligned}
& \dot{x}(t)=\left[\begin{array}{cccc}
1.38 & -0.20 & 6.71 & -5.67 \\
-0.58 & -4.29 & 0 & 0.67 \\
1.06 & 4.27 & -6.65 & 5.89 \\
0.04 & 4.27 & 1.34 & -2.10
\end{array}\right] x(t)+\left[\begin{array}{cc}
0 & 0 \\
5.67 & 0 \\
1.13 & -3.14 \\
1.13 & 0
\end{array}\right] u(t)+w(t), \\
& u(t)=-\left[\begin{array}{llll}
-0.1006 & 0.2469 & 0.0952 & 0.2447 \\
-1.4099 & 0.1966 & -0.0139 & -0.0823
\end{array}\right] x\left(t_{k}\right) .
\end{aligned}
$$

We will use the polytopic description presented in Section 4.1 , with a polynomial approximation degree $N=5$ in all the results that are presented.

\subsection{Simulation results}

Here, we consider a time-step $\Delta=0.005 s$, a desired decayrate $\alpha=0.1$, and an upper-bound $W=0.01$ on the perturbations (i.e. such that $\|w(t)\| \leq 0.1\left\|x\left(t_{k}\right)\right\|$ ).

First, we apply the algorithm proposed in Section 4.3 to enlarge the lower-bound $\tau^{*}$ of the sampling function. We obtain $\tau^{*}=3 \Delta=0.015 \mathrm{~s}$, with the parameters $P=10^{-3}\left[\begin{array}{cccc}0.3608 & 0.0580 & 0.2611 & -0.1701 \\ 0.0580 & 0.2443 & 0.1029 & 0.1101 \\ 0.2611 & 0.1029 & 0.2854 & -0.1314 \\ -0.1701 & 0.1101 & -0.1314 & 0.2861\end{array}\right], \varepsilon=0.0285, \eta=$ 0.0254 , and $\mu=0.3614$.

Then, we design the sampling function (which defines the self-triggered controller) as proposed in Section 4.2.

Figure 1 presents the simulation results obtained with the designed self-triggered controller and a perturbation satisfying $\|w(t)\| \leq 0.1\left\|x\left(t_{k}\right)\right\|$.

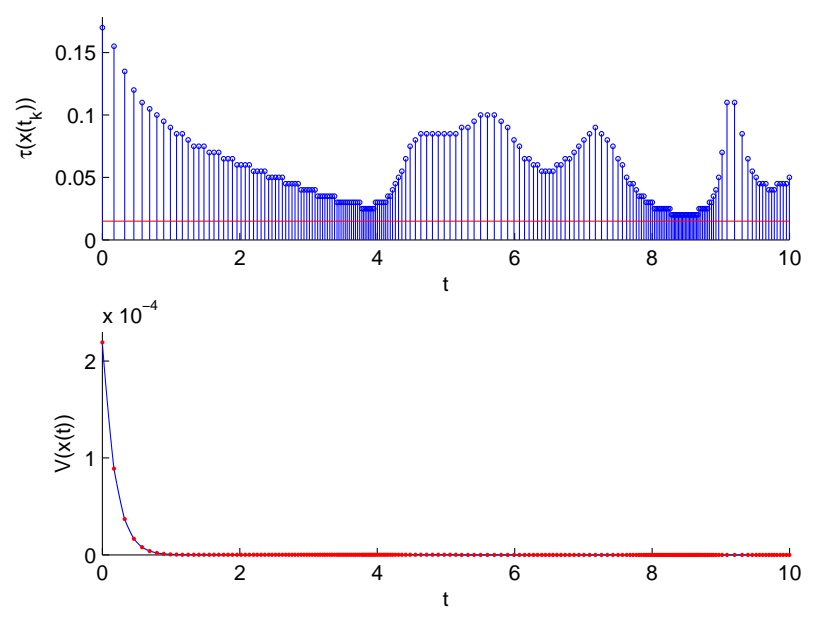

Fig. 1. Inter-execution times $\tau\left(x\left(t_{k}\right)\right)$ and Lyapunov function $V(x)=x^{T} P x$ evolution

During this $10 \mathrm{~s}$ simulation, the average sampling interval is $\tau_{\text {average }}=0.051 \mathrm{~s}$, which is more than three times the value of the optimized lower-bound $\tau^{*}$. 


\subsection{Advantages of the sampling function's lower-bound optimization}

In order to illustrate the interest of the sampling function's lower-bound optimization, we consider the simpler case of the batch reactor system without perturbation nor desired decay-rate, with the same time-step $\Delta=0.005 \mathrm{~s}$.

Using the optimization algorithm from Section 4.3, we get a lower-bound of the sampling function $\tau^{*}=36 \Delta=0.18 \mathrm{~s}$, whereas with a regular approach to compute the Lyapunov function without taking into account the sampling (for example by solving the LMI $(A-B K)^{T} P+P(A-B K) \prec$ 0 , as in Mazo Jr. et al. [2009]), we get $\tau^{*}=3 \Delta=0.015 \mathrm{~s}$.

Optimizing the lower-bound of the sampling function provides three main advantages.

1) It guarantees a large lower-bound for the sampling intervals ( $0.18 \mathrm{~s}$ instead of $0.015 \mathrm{~s}$ in this case).

2) It allows for using larger time-steps if one needs to reduce the number of online computations (in the present case, we can fix $\Delta$ up to $0.18 s$ with the optimization, and up to $0.015 s$ without).

3) It allows for reducing the number of online computations, since it is not necessary to check the condition $x^{T} \Pi_{i}^{(j)}(\Delta) x \leq 0$ for $j \in\left\{0, \cdots, j^{*}-1\right\}$ (here, it allows for saving $33 n(n+1)(N+1)$ additions and $33(n+1)(n-$ 1) $(N+1)$ multiplications during each sampling interval... provided that the sampling interval in the non-optimized case is not smaller than $36 \Delta=0.18 s$ ).

\section{CONCLUSION}

We have introduced a novel self-triggered control approach based on convex embeddings that ensures the exponential stability with a desired decay-rate for perturbed LTI systems with linear state feedback. An interesting feature of the approach is that it allows for enlarging the lowerbound of the sampling function, by computing an appropriate Lyapunov function, thanks to LMIs. Extensions to nonlinear systems are currently under study.

\section{REFERENCES}

A. Anta and P. Tabuada. To sample or not to sample: selftriggered control for nonlinear systems. IEEE Transactions on Automatic Control, 55(9):2030-2042, 2010.

Y. Cao, Y. Sun, and C. Cheng. Delay dependent robust stabilization of uncertain systems with multiple state delays. IEEE Transactions on Automatic Control, 43 (11):1608-1612, 1998.

C. Fiter, L. Hetel, W. Perruquetti, and J.-P. Richard. A state dependent sampling for linear state feedback. Automatica, 48(8):1860-1867, 2012.

C. Fiter, L. Hetel, W. Perruquetti, and J.-P. Richard. A robust polytopic approach for state-dependent sampling. In 12th European Control Conference, Zurich, Switzerland, 2013.

E. Fridman. A refined input delay approach to sampleddata control. Automatica, 46(2):421-427, 2010.

H. Fujioka. Stability analysis of systems with aperiodic sample-and-hold devices. Automatica, 45(3):771-775, 2009.

K. Gu, V. Kharitonov, and J. Chen. Stability of time-delay systems. Boston: Birkhauser, 2003.
W.P.M.H. Heemels, M.C.F Donkers, and A.R. Teel. Periodic event-triggered control based on state feedback. In 50th IEEE Conference on Decision and Control, pages 2571-2576, Orlando, Florida, USA, 2011.

L. Hetel, J. Daafouz, and C. Iung. Stabilization of arbitrary switched linear systems with unknown timevarying delays. IEEE Transactions on Automatic Control, 51(10):1668-1674, 2006.

K. Liu and E. Fridman. Wirtinger's inequality and Lyapunov-based sampled-data stabilization. Automatica, 48(1):102-108, 2012.

C.-V Loan. The sensitivity of the matrix exponential. SIAM Journal on Numerical Analysis, 14(6):971-981, 1977.

J. Lunze and D. Lehmann. A state-feedback approach to event-based control. Automatica, 46(1):211-215, 2010.

M. Mazo Jr., A. Anta, and P. Tabuada. On self-triggered control for linear systems: guarantees and complexity. In European Control Conference, Budapest, Hungary, 2009.

M. Mazo Jr., A. Anta, and P. Tabuada. An ISS selftriggered implementation of linear controllers. Automatica, 46(8):1310-1314, 2010.

R. Postoyan, A. Anta, D. Nesic, and P. Tabuada. A unifying Lyapunov-based framework for the event-triggered control of nonlinear systems. In 50th IEEE Conference on Decision and Control, pages 2559-2564, Orlando, Florida, USA, 2011.

A. Seuret. A novel stability analysis of linear systems under asynchronous samplings. Automatica, 48(1):177$182,2012$.

P. Tabuada. Event-triggered real-time scheduling of stabilizing control tasks. IEEE Transactions on Automatic Control, 52(9):1680-1685, 2007.

X. Wang and M.-D. Lemmon. Self-triggering under stateindependent disturbances. IEEE Transactions on Automatic Control, 55(6):1494-1500, 2010.

\section{Appendix A. LEMMA AND PROOFS}

\section{A.1 Lemma}

Lemma 5. (Adapted from Fiter et al. [2013]).

Consider a scalar $\alpha>0$ and a sampling function $\tau: \mathbb{R}^{n} \rightarrow$ $\mathbb{R}_{+}$. If there exists a matrix $P \in \mathbb{S}_{n}^{+*}$ such that for all $x \in \mathbb{R}^{n}$, and all $\sigma \in[0, \tau(x)]$,

$$
\left[\begin{array}{c}
\Lambda(\sigma) x+J_{w}(\sigma) \\
x \\
w(\sigma)
\end{array}\right]^{T} \Omega\left[\begin{array}{c}
\Lambda(\sigma) x+J_{w}(\sigma) \\
x \\
w(\sigma)
\end{array}\right] \leq 0
$$

with the matrices

$$
\begin{gathered}
\Lambda(\sigma)=I+\int_{0}^{\sigma} \mathrm{e}^{s A} d s(A-B K), \\
J_{w}(\sigma)=\int_{0}^{\sigma} \mathrm{e}^{A(\sigma-s)} E w(s) d s,
\end{gathered}
$$

and

$$
\Omega=\left[\begin{array}{ccc}
A^{T} P+P A+2 \alpha P & -P B K & P E \\
* & 0 & 0 \\
* & * & 0
\end{array}\right],
$$

then the system $\mathcal{S}$ is globally $\alpha$-stable.

Proof. The proof consists in rewriting the condition (5) in Proposition 1 as 
$\left[\begin{array}{c}\varphi_{\tau, w}(\sigma, x) \\ x \\ w(\sigma)\end{array}\right]^{T}\left[\begin{array}{ccc}A^{T} P+P A+2 \alpha P & -P B K & P E \\ * & 0 & 0 \\ * & * & 0\end{array}\right]\left[\begin{array}{c}\varphi_{\tau, w}(\sigma, x) \\ x \\ w(\sigma)\end{array}\right] \leq 0$

where, $x$ represents the sampled-state $x\left(t_{k}\right), \sigma$ represents the time $t-t_{k}$, and $\varphi_{\tau, w}(\sigma, x)=\Lambda(\sigma) x+J_{w}(\sigma)$ is the solution $x(t)$ of $\mathcal{S}$ with initial value $x$ and perturbation $w$.

\section{A.2 Proofs}

Proof. [Theorem 2] This proof is based on the results from Lemma 5, in the appendix. The idea is to find an upper-bound independent of the perturbation $w$ for the left part of equation (A.1) in Lemma 5 .

Consider a matrix $P \in \mathbb{S}_{n}^{+*}$, scalars $\alpha>0$ and $W \geq 0$, and a sampling function $\tau: \mathbb{R}^{n} \rightarrow \mathbb{R}_{+}$. Using the notations

$$
\begin{gathered}
M_{1}=A^{T} P+P A+2 \alpha P, M_{2}(\sigma)=-P B K+M_{1} \Lambda(\sigma), \\
M_{3}=P E, M_{4}(\sigma)=E^{T} P^{T} \Lambda(\sigma),
\end{gathered}
$$

we can rewrite the left part of equation (A.1) as

$$
\begin{aligned}
& G_{w}(\sigma, x)= \\
& x^{T}\left(\Lambda(\sigma)^{T} M_{1} \Lambda(\sigma)-\Lambda(\sigma)^{T} P B K-K^{T} B^{T} P \Lambda(\sigma)\right) x \\
& +J_{w}(\sigma)^{T} M_{1} J_{w}(\sigma)+J_{w}(\sigma)^{T} M_{2}(\sigma) x+x^{T} M_{2}(\sigma)^{T} J_{w}(\sigma) \\
& +J_{w}(\sigma)^{T} M_{3} w(\sigma)+w(\sigma)^{T} M_{3}^{T} J_{w}(\sigma) \\
& +w(\sigma)^{T} M_{4}(\sigma) x+x^{T} M_{4}(\sigma)^{T} w(\sigma) .
\end{aligned}
$$

Then, using inequality $(6)$, we get

$$
\begin{aligned}
& J_{w}(\sigma)^{T} M_{2}(\sigma) x+x^{T} M_{2}(\sigma)^{T} J_{w}(\sigma) \\
& \leq J_{w}(\sigma)^{T} \varepsilon^{-1}\left[P+A^{T} P A\right] J_{w}(\sigma)+x^{T}\left[\Lambda(\sigma)^{T} \varepsilon P \Lambda(\sigma)\right] x \\
& \quad+x^{T} \varepsilon\left[[-B K+(A+2 \alpha I) \Lambda(\sigma)]^{T} P\right. \\
& \quad[-B K+(A+2 \alpha I) \Lambda(\sigma)]] x, \\
& J_{w}(\sigma)^{T} M_{3} w(\sigma)+w(\sigma)^{T} M_{3}^{T} J_{w}(\sigma) \\
& \leq J_{w}(\sigma)^{T} P J_{w}(\sigma)+w(\sigma)^{T} E^{T} P E w(\sigma), \\
& w(\sigma)^{T} M_{4}(\sigma) x+x^{T} M_{4}(\sigma)^{T} w(\sigma) \\
& \leq w(\sigma)^{T} \varepsilon^{-1} E^{T} P E w(\sigma)+x^{T} \Lambda(\sigma)^{T} \varepsilon P \Lambda(\sigma) x, \\
& \text { and thus obtain }\left(\text { with matrices } Q_{i} \operatorname{defined~in~}(11)\right): \\
& G_{w}(\sigma, x) \leq x^{T}\left[\Lambda(\sigma)^{T} Q_{1} \Lambda(\sigma)\right. \\
& \left.\quad+\Lambda(\sigma)^{T} Q_{2}+Q_{2}^{T} \Lambda(\sigma)+\varepsilon K^{T} B^{T} P B K\right] x \\
& \quad+w(\sigma)^{T}\left(1+\varepsilon^{-1}\right) E^{T} P E w(\sigma)+J_{w}(\sigma)^{T} Q_{5} J_{w}(\sigma) .
\end{aligned}
$$

Using (7) and (4), we get

$$
w(\sigma)^{T}\left(1+\varepsilon^{-1}\right) E^{T} P E w(\sigma) \leq \eta w(\sigma)^{T} w(\sigma) \leq W \eta x^{T} x .
$$

From (7), we also have

$$
J_{w}(\sigma)^{T} Q_{5} J_{w}(\sigma) \leq \mu\left(\int_{0}^{\sigma} \mathrm{e}^{A(\sigma-s)} E w(s) d s\right)^{T}\left(\int_{0}^{\sigma} \mathrm{e}^{A(\sigma-s)} E w(s) d s\right) .
$$

Then, using Jensen's inequality (Gu et al. [2003], Proposition B.8), the inequality (2.2) in Loan [1977], along with classic inequalities and assumption (4), one gets

$$
\begin{aligned}
& J_{w}(\sigma)^{T} Q_{5} J_{w}(\sigma) \\
& \quad \leq \sigma \mu \int_{0}^{\sigma} w(s)^{T} E^{T}\left(\mathrm{e}^{A(\sigma-s)}\right)^{T}\left(\mathrm{e}^{A(\sigma-s)}\right) E w(s) d s \\
& \quad \leq \sigma \mu \int_{0}^{\sigma} \mathrm{e}^{(\sigma-s) \lambda_{\max }\left(A+A^{T}\right)} w(s)^{T} E^{T} E w(s) d s \\
& \quad \leq \sigma \mu \lambda_{\max }\left(E^{T} E\right) \int_{0}^{\sigma} \mathrm{e}^{(\sigma-s) \lambda_{\max }\left(A+A^{T}\right)}\|w(s)\|^{2} d s \\
& \quad \leq \sigma W \mu \lambda_{\max }\left(E^{T} E\right)\left(\int_{0}^{\sigma} \mathrm{e}^{\lambda_{\max }\left(A+A^{T}\right) s} d s\right)\|x\|^{2}
\end{aligned}
$$

$$
=\sigma W \mu \lambda_{\max }\left(E^{T} E\right) f_{A}(\sigma) x^{T} x
$$

with $f_{A}(\sigma)$ defined in (12). Implementing inequalities (A.6) and (A.7) in (A.5) shows that $G_{w}(\sigma, x) \leq x^{T} \Pi(\sigma) x$, with $\Pi(\sigma)$ defined in $(9)$, and thus that Lemma 5 stability conditions are satisfied if $x^{T} \Pi(\sigma) x \leq 0$ for all $x \in \mathbb{R}^{n}$ and $\sigma \in[0, \tau(x)]$.

Proof. [Theorem 3] Let $\Delta \in \mathbb{R}_{+}^{*}, j \in \mathbb{N}$, and $x \in \mathbb{R}^{n}$.

Step (1): Compute the Taylor expansion of the matrix function $\Pi$ over the time interval $[j \Delta,(j+1) \Delta]$. Remember that $\Pi(\sigma)$ involves the term $\Lambda(\sigma)$ defined in (10). Using the property

$$
\int_{0}^{a+b} \mathrm{e}^{A s} d s=\int_{0}^{a} \mathrm{e}^{A s} d s+\int_{0}^{b} \mathrm{e}^{A s} d s\left(A \int_{0}^{a} \mathrm{e}^{A s} d s+I\right),
$$

which is valid for any scalars $a$ and $b$, we can rewrite $\Lambda(\sigma)$ as a function of $\sigma^{\prime}=\sigma-j \Delta \in[0, \Delta]$ :

$$
\begin{aligned}
\Lambda(\sigma) & =I+\left(N_{j}(\Delta)+\int_{0}^{\sigma^{\prime}} \mathrm{e}^{A s} d s N_{j}^{\prime}(\Delta)\right)(A-B K) \\
& =\Gamma_{1}^{(j)}(\Delta)+\sum_{i=1}^{\infty} \frac{A^{i-1}}{i !} \sigma^{i} \Gamma_{2}^{(j)}(\Delta),
\end{aligned}
$$

with the notations from (17). Using this last equation, one can show that (the computational details are omitted)

$$
\Pi(\sigma)=\sum_{k=0}^{\infty} \Psi_{k}^{(j)}(\Delta) \sigma^{\prime k},
$$

with the matrices $\Psi_{k}^{(j)}(\Delta)$ defined in (16). Here, the matrices $L_{k}^{(j)}(\Delta)$ that appear in (16) come from the Taylor expansion of the term $Q_{4}(\sigma)=\sigma W \mu \lambda_{\max }\left(E^{T} E\right) f_{A}(\sigma) I$, and are defined in (18) and (19).

A polynomial approximation of order $N$ of $\Pi$ on the interval $[j \Delta,(j+1) \Delta]$ can therefore be expressed as

$$
\tilde{\Pi}_{N}^{(\Delta, j)}(\sigma)=\sum_{k=0}^{N} \Psi_{k}^{(j)}(\Delta) \sigma^{\prime k}
$$

Step (2): The approximation error term $R_{N}^{(\Delta, j)}(\sigma)=$

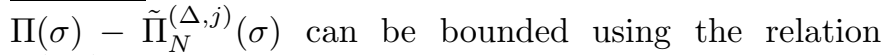
$R_{N}^{(\Delta, j)}(\sigma) \preceq \nu^{(j)}(\Delta) I$, with $\nu^{(j)}(\Delta)$ defined in (20). With this, it is clear that if $x^{T}\left(\tilde{\Pi}_{N}^{(\Delta, j)}(\sigma)+\nu^{(j)}(\Delta) I\right) x \leq 0$, then $x^{T} \Delta(\sigma) x \leq 0$.

Step (3): Since the function

$$
x^{T}\left(\tilde{\Pi}_{N}^{(\Delta, j)}(.)+\nu^{(j)}(\Delta) I\right) x:[j \Delta,(j+1) \Delta] \rightarrow \mathbb{R}
$$

is polynomial, we can use the convex embedding design from Hetel et al. [2006], Section 3, to prove that if we have $x^{T} \Pi_{i}^{(j)}(\Delta) x \leq 0$ for all $i \in\{0, \cdots, N\}$, with $\Pi_{i}^{(j)}(\Delta)=\left(\sum_{k=0}^{i} \Psi_{k}^{(j)}(\Delta) \Delta^{k}\right)+\nu^{(j)}(\Delta) I$, then we have $x^{T}\left(\tilde{\Pi}_{N}^{(\Delta, j)}(\sigma)+\nu^{(j)}(\Delta) I\right) x \leq 0$ for all $\sigma \in[j \Delta,(j+1) \Delta]$, and therefore $\Pi(\sigma) \leq 0$.

Proof. [Theorem 4] The matrix inequalities and Theorem 3 ensure that the stability conditions of Theorem 2 are satisfied for the sampling function $\bar{\tau}(x)=\max \left(\tau(x), j^{*} \Delta\right)$. 